\title{
EXACT AND APPROXIMATE SOLUTIONS \\ OF DELAY DIFFERENTIAL EQUATIONS \\ WITH NONLOCAL HISTORY CONDITIONS
}

S. AGARWAL AND D. BAHUGUNA

Received 28 April 2004 and in revised form 23 July 2004

We study the exact and approximate solutions of a delay differential equation with various types of nonlocal history conditions. We establish the existence and uniqueness of mild, strong, and classical solutions for a class of such problems using the method of semidiscretization in time. We also establish a result concerning the global existence of solutions. Finally, we consider some examples and discuss their exact and approximate solutions.

\section{Introduction}

We are concerned here with exact and approximate solutions of the following delay differential equation:

$$
\begin{gathered}
\frac{\partial w}{\partial t}(x, t)-\frac{\partial^{2} w}{\partial x^{2}}(x, t)=f(x, t, w(x, t), w(x, t-\tau)), \quad 0<t \leq T<\infty, x \in(a, b), \\
w(a, t)=w(b, t)=0, \quad t \geq 0, \\
g\left(w_{[-\tau, 0]}\right)=\phi,
\end{gathered}
$$

where the sought-for real-valued function $w$ is defined on $(a, b) \times[-\tau, T], \tau>0, a<b, f$ is a smooth real-valued function defined on $(a, b) \times[0, T] \times \mathbb{R}^{2}, g$ is a map from $\mathscr{C}_{0}:=$ $C\left([-\tau, 0] ; L^{2}(a, b)\right)$ into $L^{2}(a, b), w_{[-\tau, 0]}$ is the restriction of $w$ on $(a, b) \times[-\tau, 0]$, and $\phi \in L^{2}(a, b)$.

Some of the cases of the nonlocal history function $g$ in which we will be interested are the following.

(I) $g(\psi)(x)=\int_{-\tau}^{0} k(s) \psi(s)(x) d s$ for $x \in(a, b)$ and $\psi \in \mathscr{C}_{0}$, where $k \in L^{1}(-\tau, 0)$ with $\kappa:=\int_{-\tau}^{0} k(s) d s \neq 0$.

(II) $g(\psi)(x)=\sum_{i=1}^{n} c_{i} \psi\left(\theta_{i}\right)(x)$ for $x \in(a, b)$ and $\psi \in \mathscr{C}_{0}$, where $-\tau \leq \theta_{1}<\theta_{2}<\cdots<$ $\theta_{n} \leq 0$ and $C:=\sum_{i=1}^{n} c_{i} \neq 0$.

(III) $g(\psi)(x)=\sum_{i=1}^{n}\left(c_{i} / \epsilon_{i}\right) \int_{\theta_{i}-\epsilon_{i}}^{\theta_{i}} \psi(s)(x) d s$ for $x \in(a, b)$ and $\psi \in \mathscr{C}_{0}$, where $\theta_{i}$ and $c_{i}$ are as in (II) and $\epsilon_{i}>0$ for $i=1,2, \ldots, n$. 
Nonlocal abstract differential and functional differential equations have been extensively studied in the literature. We refer to the works of Byszewski [6], Byszewski and Lakshmikantham [8], Balachandran and Chandrasekaran [5], and Lin and Liu [11]. Most of them used semigroup theory and fixed point theorem to establish the unique existence and regularity of solution. In [7], Byszewski and Akca applied Schauder's fixed point principle to prove the theorems for existence of mild and classical solutions of nonlocal Cauchy problem of the form

$$
\begin{gathered}
u^{\prime}(t)+A u(t)=f\left(t, u(t), u\left(b_{1}(t)\right), \ldots, u\left(b_{m}(t)\right)\right), \quad t \in(0, T], \\
u(0)+g(u)=u_{0},
\end{gathered}
$$

where $-A$ is the infinitesimal generator of a compact $C_{0}$ semigroup in a Banach space.

In our recent work $[1,2]$, we studied the functional differential equation (1.2) with the nonlocal history condition $h\left(u_{[-\tau, 0]}\right)=\phi$, where $h$ is a Volterra-type operator from $\mathscr{C}_{0}$ into itself and $\phi \in \mathscr{C}_{0}$. We made use of method of semidiscretization in time to derive the existence and uniqueness of a strong solution. Many authors have used and developed the method of semidiscretization for nonlinear evolution and nonlinear functional evolution equations, see, for instance, the papers of Kartsatos and Parrott [9], Kartsatos and Zigler [10], Bahuguna and Raghavendra [4], and the references listed therein.

Our purpose here is to study the exact and approximate solutions of the delay differential equation (1.1) with a nonlocal condition. In doing so, we first use the method of semidiscretization to derive the existence of a unique strong solution, then we prove that strong solution is a classical solution if additional conditions are assumed on the operator. The global existence of a solution for (1.1), a nonconsidered problem in $[1,2]$, is also established with an additional assumption (see Theorem 4.1). The result of the paper consists, among other things, in that we obtain a solution of problem of much stronger regularity than in $[1,2]$.

\section{Existence and uniqueness of solutions}

The existence and uniqueness results have been established for the more general case of (2.2) in Bahuguna [3]. For the sake of completeness, we briefly mention the ideas and the main result of the existence and uniqueness.

If we take $H:=L^{2}(a, b)$, the real Hilbert space of all real-valued square-integrable functions on the interval $(a, b)$, and the linear operator $A$ defined by

$$
D(A):=\left\{u \in H: u^{\prime \prime} \in H, u(a)=u(b)=0\right\}, \quad A u=-u^{\prime \prime}
$$

then it is well known that $-A$ generates an analytic semigroup $e^{t A}, t \geq 0$, in $H$. If we define $u:[-\tau, T] \rightarrow H$ given by $u(t)(x)=w(x, t)$, then (1.1) may be rewritten as the following evolution equation:

$$
\begin{aligned}
u^{\prime}(t)+A u(t)= & F(t, u(t), u(t-\tau)), \quad 0<t \leq T, \\
& h\left(u_{[-\tau, 0]}\right)=\Phi,
\end{aligned}
$$


for a suitably defined function $F:[0, T] \times H^{2} \rightarrow H, 0<T<\infty, \Phi \in \mathscr{C}_{0}:=C([-\tau, 0] ; H)$, the linear operator $A$, defined from the domain $D(A) \subset H$ into $H$, is such that $-A$ is the infinitesimal generator of a $C_{0}$ semigroup $S(t), t \geq 0$, of contractions in $H$, the map $h$ is defined from $\mathscr{C}_{0}$ into $\mathscr{C}_{0}$. Here $\mathscr{C}_{t}:=C([-\tau, t] ; H)$ for $t \in[0, T]$ is the space of all continuous functions from $[-\tau, t]$ into $H$ endowed with supremum norm

$$
\|\psi\|_{t}=\sup _{-\tau \leq \eta \leq t}\|\psi(\eta)\|, \quad \psi \in \mathscr{C}_{t} .
$$

Suppose that there exists a $\chi \in \mathscr{C}_{0}$ such that $h(\chi)=\Phi$. Let $\tilde{T}$ be any number such that $0<\widetilde{T} \leq T$. A function $u \in \mathscr{C}_{\widetilde{T}}$ such that

$$
u(t)= \begin{cases}\chi(t), & t \in[-\tau, 0] \\ S(t) \chi(0)+\int_{0}^{t} S(t-s) F(s, u(s), u(s-\tau)) d s, & t \in[0, \widetilde{T}]\end{cases}
$$

is called a mild solution of (2.2) on $[-\tau, \tilde{T}]$. By a strong solution $u$ of $(2.2)$ on $[-\tau, \tilde{T}]$, we mean a function $u \in \mathscr{C}_{\tilde{T}}$ such that $u(t) \in D(A)$ for a.e. $t \in[0, \widetilde{T}], u$ is differentiable a.e. on $[0, \tilde{T}]$ and

$$
u^{\prime}(t)+A u(t)=F(t, u(t), u(t-\tau)), \quad \text { a.e. } t \in[0, \tilde{T}]
$$

A mild solution $u$ of $(1.1)$ on $[-\tau, \tilde{T}]$ is called a classical solution of $(1.1)$ if $u(t) \in D(A)$ for all $t \in(0, \tilde{T}]$ and $u \in C^{1}((0, \tilde{T}) ; H)$, and

$$
u^{\prime}(t)+A u(t)=F(t, u(t), u(t-\tau)), \quad t \in(0, \tilde{T}] .
$$

We have the following existence and uniqueness result for (2.2).

Theorem 2.1. Suppose that there exists a Lipschitz continuous $\chi \in \mathscr{C}_{0}$ such that $h(\chi)=\Phi$ and $F$ satisfies the condition

$$
\left\|F\left(t_{1}, u_{1}, v_{1}\right)-F\left(t_{2}, u_{2}, v_{2}\right)\right\| \leq L_{F}(r)\left[\left|t_{1}-t_{2}\right|+\left\|u_{1}-u_{2}\right\|+\left\|v_{1}-v_{2}\right\|\right]
$$

for all $t_{i} \in[0, T], u_{i}, v_{i} \in B_{r}(H, \chi(0)), i=1,2$, where $B_{r}\left(Z, z_{0}\right)$ denotes the closed ball of radius $r>0$ centered at $z_{0}$ in the Banach space $Z$. Then there exists a strong solution $u$ of (2.2) either on the whole interval $[-\tau, T]$ or on a maximal interval $\left[-\tau, t_{\max }\right), 0<t_{\max } \leq T$, such that $u$ is a strong solution of (2.2) on $[-\tau, \tilde{T}]$ for every $0<\widetilde{T}<t_{\max }$, and in the latter case,

$$
\lim _{t \rightarrow t_{\max }-}\|u(t)\|=\infty
$$

If, in addition, $S(t)$ is an analytic semigroup in $H$, then $u$ is a classical Lipschitz continuous solution on every compact subinterval of the interval of existence. Furthermore, $u$ is unique in $\left\{\psi \in \mathscr{C}_{\widetilde{T}}: \psi=\chi\right.$ on $\left.[-\tau, 0]\right\}$ for every compact subinterval $[-\tau, \widetilde{T}]$ of the interval of existence. 


\section{Nonlocal history-valued delay differential equations}

\section{Approximations}

In this section, we consider the application of the method of semidiscretization in time and the convergence of the approximate solutions. We first establish the existence and uniqueness of a strong solution of (2.2) for any given $\chi \in \mathscr{C}_{0}$ and $\chi(0) \in D(A)$. Fix $R>0$ and let $R_{0}:=R+\sup _{t \in[-\tau, 0]}\|\chi(t)-\chi(0)\|$. We choose $t_{0}$ such that

$$
0<t_{0} \leq T, \quad t_{0} M_{0} \leq R,
$$

where, $M_{0}:=\|A \chi(0)\|+L_{f}\left(R_{0}\right)\left(T+5 R_{0}\right)+\|f(0, \chi(0), \chi(0))\|$.

For $n \in \mathbb{N}$, let $h_{n}=t_{0} / n$. We set $u_{0}^{n}=\chi(0)$ for all $n \in \mathbb{N}$ and define each of $\left\{u_{j}^{n}\right\}_{j=1}^{n}$ as the unique solution of the equation

$$
\frac{u-u_{j-1}^{n}}{h_{n}}+A u=F\left(t_{j}^{n}, u_{j-1}^{n}, \widetilde{u}_{j-1}^{n}\left(t_{j}^{n}-\tau\right)\right),
$$

where $\tilde{u}_{0}^{n}(t)=\chi(t)$ for $t \in[-\tau, 0], \tilde{u}_{0}^{n}(t)=\chi(0)$ for $t \in\left[0, t_{1}^{n}\right]$, and for $2 \leq j \leq n$,

$$
\tilde{u}_{j-1}^{n}(t)= \begin{cases}\chi(t), & t \in[-\tau, 0], \\ u_{i-1}^{n}+\frac{1}{h_{n}}\left(t-t_{i-1}^{n}\right)\left(u_{i}^{n}-u_{i-1}^{n}\right), & t \in\left[t_{i-1}^{n}, t_{i}^{n}\right], i=1,2, \ldots, j-1, \\ u_{j-1}^{n}, & t \in\left[t_{j-1}^{n}, t_{j}^{n}\right] .\end{cases}
$$

The existence of a unique $u_{j}^{n} \in D(A)$ satisfying (3.2) is a consequence of the $m$-monotonicity of $A$. We define the sequence $\left\{U^{n}\right\} \subset \mathscr{C}_{t_{0}}$ of polygonal functions

$$
U^{n}(t)= \begin{cases}\chi(t), & t \in[-\tau, 0], \\ u_{j-1}^{n}+\frac{1}{h_{n}}\left(t-t_{j-1}^{n}\right)\left(u_{j}^{n}-u_{j-1}^{n}\right), & t \in\left(t_{j-1}^{n}, t_{j}^{n}\right],\end{cases}
$$

and prove the convergence of $\left\{U^{n}\right\}$ to a unique strong solution $u$ of $(2.2)$ as $n \rightarrow \infty$. Before proving the convergence, we state and prove some lemmas which will be used to establish the main result.

Lemma 3.1. For $n \in \mathbb{N}, j=1,2, \ldots, n$,

$$
\left\|u_{j}^{n}-\chi(0)\right\| \leq R
$$

Proof. From (3.2) for $j=1$, we have

$$
\left\|u_{1}^{n}-\chi(0)\right\| \leq h_{n} M_{0} \leq R .
$$


Assume that $\left\|u_{i}^{n}-\chi(0)\right\| \leq R$ for $i=1,2, \ldots, j-1$. Now, for $2 \leq j \leq n$,

$$
\left\|u_{j}^{n}-\chi(0)\right\| \leq\left\|u_{j-1}^{n}-\chi(0)\right\|+h_{n} M_{0} .
$$

Repeating the above inequality, we obtain

$$
\left\|u_{j}^{n}-\chi(0)\right\| \leq j h_{n} M_{0} \leq R,
$$

as $j h_{n} \leq t_{0}$ for $0 \leq j \leq n$. This completes the proof of the lemma.

Lemma 3.2. There exists a positive constant $K$ independent of the discretization parameters $n, j$, and $h_{n}$ such that

$$
\left\|\frac{u_{j}^{n}-u_{j-1}^{n}}{h_{n}}\right\| \leq K, \quad j=1,2, \ldots, n, n=1,2, \ldots
$$

Proof. In this proof and subsequently, $K$ will represent a generic constant independent of $j, h_{n}$, and $n$. From (3.2), for $j=1$ and monotonicity of $A$, we have

$$
\left\|\frac{u_{1}^{n}-u_{0}^{n}}{h_{n}}\right\| \leq M_{0} \leq K
$$

Now, for $2 \leq j \leq n$, using monotonicity of $A$ and local Lipschitz-like condition (2.7) of $F$, we get

$$
\max _{\{1 \leq k \leq j\}}\left\|\frac{u_{k}^{n}-u_{k-1}^{n}}{h_{n}}\right\| \leq\left(1+C h_{n}\right) \max _{\{1 \leq k \leq j-1\}}\left\|\frac{u_{k}^{n}-u_{k-1}^{n}}{h_{n}}\right\|+C h_{n}
$$

where $C$ is a positive constant independent of $j, h_{n}$, and $n$. Repeating the above inequality, we obtain

$$
\left\|\frac{u_{j}^{n}-u_{j-1}^{n}}{h_{n}}\right\| \leq K
$$

This completes the proof of the lemma.

We introduce another sequence $\left\{X^{n}\right\}$ of step functions from $\left[-h_{n}, t_{0}\right]$ into $H$ by

$$
X^{n}(t)= \begin{cases}\chi(0), & t \in\left[-h_{n}, 0\right] \\ u_{j}^{n}, & t \in\left(t_{j-1}^{n}, t_{j}^{n}\right]\end{cases}
$$

For notational convenience, let

$$
f^{n}(t)=f\left(t_{j}^{n}, u_{j-1}^{n}, \tilde{u}_{j-1}^{n}\left(t_{j}^{n}-\tau\right)\right), \quad t \in\left(t_{j-1}^{n}, t_{j}^{n}\right], 1 \leq j \leq n .
$$


Then (3.2) may be rewritten as

$$
\frac{d^{-}}{d t} U^{n}(t)+A X^{n}(t)=f^{n}(t), \quad t \in\left(0, t_{0}\right]
$$

where $d^{-} / d t$ denotes the left derivative in $\left(0, t_{0}\right]$. Also, for $t \in\left(0, t_{0}\right]$, we have

$$
\int_{0}^{t} A X^{n}(s) d s=\chi(0)-U^{n}(t)+\int_{0}^{t} f^{n}(s) d s .
$$

Next, we prove the convergence of $U^{n}$ to $u$ in $\mathscr{C}_{t_{0}}$.

Lemma 3.3. There exists $u \in \mathscr{C}_{t_{0}}$ such that $U^{n} \rightarrow u$ in $\mathscr{C}_{t_{0}}$ as $n \rightarrow \infty$. Moreover, $u$ is Lipschitz continuous on $\left[0, t_{0}\right]$.

Proof. It can be easily proved using monotonicity of $A$ and condition (2.7) of $F$ in (3.15) (cf. $[1,2])$.

Proof of Theorem 2.1. By proceeding as in Agarwal and Bahuguna [2] we can show the existence and uniqueness of the strong solution on $\left[-\tau, t_{0}\right]$ as well as the continuation of the solution on $[-\tau, T]$. Thus we have that there exists a strong solution of (2.2) either on the whole interval $[-\tau, T]$ or on the maximal interval of existence $\left[-\tau, t_{\max }\right), 0<t_{\max } \leq T$.

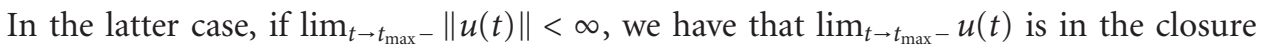
of $D(A)$ in $H$, and if it is in $D(A)$, then, following the same steps as before, $u(t)$ can be extended beyond $t_{\max }$, which contradicts the definition of the maximal interval of existence.

To prove the remaining part of Theorem 2.1, we assume the interval of existence $[-\tau$, $T]$. The proof may be modified for the interval $\left[-\tau, t_{\max }\right)$. Also $-A$ is the infinitesimal generator of $C_{0}$ semigroup. The function $\bar{F}:[0, T] \rightarrow H:=L^{2}(a, b)$ given by

$$
\bar{F}(t)=F(t, u(t), u(t-\tau))
$$

is Lipschitz continuous and therefore continuous on $[0, T]$ and $\bar{F} \in L^{1}((0, T) ; H)$. Now it is easy to see that if $u$ is the strong solution of (2.2), then $u$ is given by

$$
u(t)= \begin{cases}\chi(t), & t \in[-\tau, 0], \\ S(t) \chi(0)+\int_{0}^{t} S(t-s) \bar{F}(s) d s, & t \in[0, T]\end{cases}
$$

and therefore is a mild solution of (2.2). If $S(t)$ is an analytic semigroup in $H$, then by the use of Corollary 3.3 in Pazy [12, page 113], we obtain that $u$ is a classical solution of (2.2). Clearly, if $\chi \in \mathscr{C}_{0}$ satisfying that $h(\chi)=\Phi$ is unique on $[-\tau, 0], u$ is unique since for two $\chi, \tilde{\chi}$ in $\mathscr{C}_{0}$ satisfying $h(\chi)=h(\tilde{\chi})=\Phi$ with $\chi \neq \tilde{\chi}$, the corresponding solutions $u_{\chi}$ and $u_{\tilde{\chi}}$ belonging to $\left\{\psi \in \mathscr{C}_{\tilde{T}}: \psi=\chi\right.$ on $\left.[-\tau, 0]\right\}$ and $\left\{\psi \in \mathscr{C}_{\tilde{T}}: \psi=\tilde{\chi}\right.$ on $\left.[-\tau, 0]\right\}$, respectively, are different.

\section{Global existence}

We turn now to global existence. Here further assumptions are made, under the consideration of which, the existence of a global solution is established. 
Theorem 4.1. Let $-A$ be the infinitesimal generator of a compact $C_{0}$ semigroup $S(t), t \geq 0$, on $H$. Let $F:[0, \infty) \times H \rightarrow H$ be continuous and map bounded sets in $[0, \infty) \times H$ into bounded sets in $H$. Also there exist two locally integrable functions $k_{1}(s)$ and $k_{2}(s)$ such that

$$
\|F(s, u, v)\| \leq k_{1}(s)(\|u\|+\|v\|)+k_{2}(s), \quad \text { for } 0 \leq s<\infty, u, v \in H .
$$

Then, for every $\chi \in \mathscr{C}_{0}$ satisfying $h(\chi)=\Phi$, problem (2.2) has a global solution $u \in C([-\tau$, $\infty), H)$.

Proof. We know that the corresponding solution $u$ exists on the interval $[-\tau, T)$ and is given by

$$
u(t)= \begin{cases}\chi(t), & t \in[-\tau, 0] \\ S(t) \chi(0)+\int_{0}^{t} S(t-s) F(s, u(s), u(s-\tau)) d s, & t \in[0, T)\end{cases}
$$

We also know that $\|S(t)\| \leq M e^{\omega t}$ for some $M \geq 1$ and $\omega \geq 0$. Let

$$
\xi(t)=(M+1)\|\chi\|_{0}+\int_{0}^{t} M e^{-\omega s} k_{2}(s) d s
$$

The function $\xi$ thus defined is obviously continuous on $[0, \infty)$.

For $t \in[-\tau, 0]$,

$$
\|u(t)\| e^{-\omega t}=\|\chi(t)\| e^{-\omega t} \leq\|\chi\|_{0} \leq M\|\chi\|_{0}
$$

and for $t \in[0, T)$,

$$
\begin{aligned}
\|u(t)\| e^{-\omega t} & \leq e^{-\omega t}\|S(t) \chi(0)\|+e^{-\omega t} \int_{0}^{t}\|S(t-s) F(s, u(s), u(s-\tau))\| d s \\
& \leq M\|\chi\|_{0}+M \int_{0}^{t} e^{-\omega s} k_{1}(s)(\|u(s)\|+\|u(s-\tau)\|) d s+M \int_{0}^{t} e^{-\omega s} k_{2}(s) d s \\
& \leq M\|\chi\|_{0}+M \int_{0}^{t} e^{-\omega s} k_{2}(s) d s+2 M \int_{0}^{t} e^{-\omega s} k_{1}(s) \sup _{\eta \in[-\tau, s]}\|u(\eta)\| d s .
\end{aligned}
$$

The above inequality implies that

$$
e^{-\omega t} \sup _{\eta \in[-\tau, t]}\|u(\eta)\| \leq \xi(t)+2 M \int_{0}^{t} e^{-\omega s} k_{1}(s) \sup _{\eta \in[-\tau, s]}\|u(\eta)\| d s .
$$

By the application of Gronwall's inequality, we have

$$
e^{-\omega t} \sup _{\eta \in[-\tau, t]}\|u(\eta)\| \leq \xi(t)+2 M \int_{0}^{t} k_{1}(s) \xi(s) \exp \left\{2 M \int_{s}^{t} k_{1}(r) d r\right\} d s
$$

which implies the boundedness of $\|u(t)\|$ by a continuous function. Consequently, there exists a global solution $u$ of (2.2) (see Theorem 2.2 on page 193 in Pazy [12]). 


\section{Examples}

In this section, to illustrate the applicability of our work, we discuss the exact and approximate solutions of some initial boundary value problems.

As a first example, we consider the equation

$$
\frac{\partial u}{\partial t}(t, x)-\frac{\partial^{2} u}{\partial x^{2}}(t, x)=u(t-\tau, x)-e^{-2 t}\left(1+e^{2 \tau}\right) \sin x, \quad t>0, x \in[0, \pi]
$$

with the boundary condition

$$
u(t, 0)=u(t, \pi)=0, \quad t>0,
$$

and a nonlocal history condition

$$
\frac{1}{\tau} \int_{-\tau}^{0} e^{2 s} u(s, x) d s=\sin x, \quad x \in[0, \pi],
$$

where $\tau>1$ is arbitrary. Let $H=L^{2}([0, \pi])$. The operator $A$ with domain $D(A)=\{v \in$ $\left.H: v^{\prime \prime} \in H, v(0)=v(\pi)=0\right\}$ is given by

$$
A v=-\frac{d^{2} v}{d x^{2}} \quad \text { for } v \in D(A)
$$

Then $-A$ is the infinitesimal generator of an analytic semigroup $S(t), t \geq 0$, in $H$.

An exact solution of (5.1) is

$$
u(t, x)=e^{-2 t} \sin x, \quad t \geq-\tau, x \in[0, \pi] .
$$

In this case $\chi_{1} \in \mathscr{C}_{0}:=C\left([-\tau, 0] ; L^{2}([0, \pi])\right)$ is given by

$$
\chi_{1}(t)(x)=e^{-2 t} \sin x
$$

so that the history condition is satisfied.

Divide the interval $I=[0,1]$ into ten subintervals $I_{1}, I_{2}, \ldots, I_{10}\left(I_{j}=\left[t_{j-1}, t_{j}\right], j=\right.$ $1,2, \ldots, 10)$ of length $h=0.1$. For $t_{0}=0$, set $u_{0}(x)=\sin x$ and find, subsequently, for $t_{j}$, the approximate solutions $u_{j}, j=1,2, \ldots, 10$, so that

$$
\begin{gathered}
\frac{u_{j}(x)-u_{j-1}(x)}{h}-u_{j}^{\prime \prime}(x)=\chi_{1}\left(t_{j}-\tau\right)(x)-e^{-2 t_{j}}\left(1+e^{2 \tau}\right) \sin x \\
u_{j}(0)=u_{j}(\pi)=0
\end{gathered}
$$

that is,

$$
\begin{gathered}
\frac{u_{j}(x)-u_{j-1}(x)}{h}-u_{j}^{\prime \prime}(x)=e^{-2 t_{j}} \sin x, \\
u_{j}(0)=u_{j}(\pi)=0,
\end{gathered}
$$

is satisfied for $j=1,2, \ldots, 10$. 
Table 5.1. Approximate and exact solutions for the first example $\left(\chi=e^{-2 t} \sin x\right)$.

\begin{tabular}{lc}
\hline Approximate solution & Exact solution \\
\hline$u_{1}(x)=0.834661 \sin x$ & $u\left(x, t_{1}\right)=0.818731 \sin x$ \\
$u_{2}(x)=0.697844 \sin x$ & $u\left(x, t_{2}\right)=0.670320 \sin x$ \\
$u_{3}(x)=0.584512 \sin x$ & $u\left(x, t_{3}\right)=0.548812 \sin x$ \\
$u_{4}(x)=0.490526 \sin x$ & $u\left(x, t_{4}\right)=0.449329 \sin x$ \\
$u_{5}(x)=0.412490 \sin x$ & $u\left(x, t_{5}\right)=0.367879 \sin x$ \\
$u_{6}(x)=0.347609 \sin x$ & $u\left(x, t_{6}\right)=0.301194 \sin x$ \\
$u_{7}(x)=0.293590 \sin x$ & $u\left(x, t_{7}\right)=0.246597 \sin x$ \\
$u_{8}(x)=0.248546 \sin x$ & $u\left(x, t_{8}\right)=0.201896 \sin x$ \\
$u_{9}(x)=0.210924 \sin x$ & $u\left(x, t_{9}\right)=0.165299 \sin x$ \\
$u_{10}(x)=0.179446 \sin x$ & $u\left(x, t_{10}\right)=0.135335 \sin x$ \\
\hline
\end{tabular}

For $j=1,(5.8)$ becomes

$$
\begin{aligned}
u_{1}^{\prime \prime}(x)-\frac{1}{h} u_{1}(x) & =\left(-\frac{1}{h}+e^{-2 t_{1}}\right) \sin x \\
u_{1}(0) & =u_{1}(\pi)=0
\end{aligned}
$$

Consequently, we solve a second-order ordinary differential equation. In this case, the solution is

$$
u_{1}(x)=\frac{1}{1+h}\left(1-h e^{-2 h}\right) \sin x
$$

Similarly, for $j=2,(5.8)$ yields

$$
\begin{aligned}
u_{2}^{\prime \prime}(x)-\frac{1}{h} u_{2}(x)= & \left(-\frac{1}{h(1+h)}\left(1-h e^{-2 t_{1}}\right)+e^{-2 t_{2}}\right) \sin x \\
& u_{2}(0)=u_{2}(\pi)=0 .
\end{aligned}
$$

On solving this equation in the same way as before, we get

$$
u_{2}(x)=\frac{1}{(1+h)^{2}}\left[1-h e^{-2 h}\left(1+(1+h) e^{-2 h}\right)\right] \sin x .
$$

Similar results are easily obtained for $j=3,4, \ldots, 10$. Thus we have

$$
u_{j}(x)=\frac{1}{(1+h)^{j}}\left[1-h e^{-2 h}\left(1+(1+h) e^{-2 h}+(1+h)^{2} e^{-4 h}+\cdots+(1+h)^{j-1} e^{2(j-1) h}\right)\right] \sin x
$$

or

$$
u_{j}(x)=\frac{1}{(1+h)^{j}}\left[1-h e^{-2 h}\left(\frac{1-(1+h)^{j} e^{-2 j h}}{1-(1+h) e^{-2 h}}\right)\right] \sin x, \quad j=1,2, \ldots, 10 .
$$

Putting here $h=0.1$ and rounding off to six decimals, we finally obtain the approximate solutions $u_{j}(x)$ at $t_{j}, j=1,2, \ldots, 10$ (see Table 5.1 ). 
We also calculate the exact solution of (5.1) for $t=t_{1}=0.1, \ldots, t=t_{10}=1$ (see Table 5.1).

In the next step we choose another function

$$
\chi_{2}(t)(x)=\frac{2 \tau}{1-e^{-2 \tau}} \sin x
$$

in $\mathscr{C}_{0}$ which differs from $\chi_{1}$ and satisfies the history condition (5.3).

Divide the interval $I=[0,1]$ into the same number of subintervals with step length $h=0.1$. For $t_{0}=0$, set $u_{0}(x)=\left(2 \tau / 1-e^{-2 \tau}\right) \sin x$ and find the approximations $u_{j}$ so that

$$
\frac{u_{j}(x)-u_{j-1}(x)}{h}-u_{j}^{\prime \prime}(x)=\chi_{2}\left(t_{j}-\tau\right)(x)-e^{-2 t_{j}}\left(1+e^{2 \tau}\right) \sin x, \quad u_{j}(0)=u_{j}(\pi)=0,
$$

that is,

$$
\frac{u_{j}(x)-u_{j-1}(x)}{h}-u_{j}^{\prime \prime}(x)=\left[\frac{-2 \tau}{1-e^{-2 \tau}}+e^{-2 t_{j}}\left(1+e^{2 \tau}\right)\right] \sin x, \quad u_{j}(0)=u_{j}(\pi)=0,
$$

is fulfilled for $j=1,2, \ldots, 10$.

Following the calculations similar to the previous case, we obtain the approximate solutions $u_{j}, j=1,2, \ldots, 10$, as follows:

$$
\begin{aligned}
& u_{1}(x)=\left[\frac{\tau}{\sinh 2 \tau}-\frac{h}{(1+h)} e^{-2 h}\right]\left(1+e^{2 \tau}\right) \sin x, \\
& u_{2}(x)=\left[\frac{\tau}{\sinh 2 \tau}-\frac{h e^{-2 h}}{(1+h)^{2}}\left(1+(1+h) e^{-2 h}\right)\right]\left(1+e^{2 \tau}\right) \sin x,
\end{aligned}
$$

and

$$
u_{j}(x)=\left[\frac{\tau}{\sinh 2 \tau}-\frac{h e^{-2 h}}{(1+h)^{j}}\left(\frac{1-(1+h)^{j} e^{-2 j h}}{1-(1+h) e^{-2 h}}\right)\right]\left(1+e^{2 \tau}\right) \sin x, \quad j=1,2, \ldots, 10 .
$$

Putting here $h=0.1$ and rounding off to six decimals, we get approximate solutions which are tabulated in Table 5.2.

In this case the exact solution is obtained by solving the partial differential equation

$$
\begin{gathered}
\frac{\partial u}{\partial t}(t, x)-\frac{\partial^{2} u}{\partial x^{2}}(t, x)=\frac{2 \tau}{1-e^{-2 \tau}} \sin x-e^{-2 t}\left(1+e^{2 \tau}\right) \sin x, \quad t>0, x \in[0, \pi], \\
u(t, 0)=u(t, \pi)=0, \quad t>0, \\
u(x, 0)=\frac{2 \tau}{1-e^{-2 \tau}} \sin x, \quad x \in[0, \pi] .
\end{gathered}
$$

We take the solution of the form

$$
u(t, x)=T(t) \sin x
$$


Table 5.2. Approximate and exact solutions for the first example $\left(\chi=\left(2 \tau /\left(1-e^{-2 \tau}\right)\right) \sin x\right)$.

\begin{tabular}{ll}
\hline Approximate solution & Exact solution \\
\hline$u_{1}(x)=\left(\frac{q}{\sinh 2 q}-0.074430\right)\left(1+e^{2 q}\right) \sin x$ & $u\left(x, t_{1}\right)=\left(\frac{q}{\sinh 2 q}-0.086106\right)\left(1+e^{2 q}\right) \sin x$ \\
$u_{2}(x)=\left(\frac{q}{\sinh 2 q}-0.128602\right)\left(1+e^{2 q}\right) \sin x$ & $u\left(x, t_{2}\right)=\left(\frac{q}{\sinh 2 q}-0.148411\right)\left(1+e^{2 q}\right) \sin x$ \\
$u_{3}(x)=\left(\frac{q}{\sinh 2 q}-0.166803\right)\left(1+e^{2 q}\right) \sin x$ & $u\left(x, t_{3}\right)=\left(\frac{q}{\sinh 2 q}-0.192007\right)\left(1+e^{2 q}\right) \sin x$ \\
$u_{4}(x)=\left(\frac{q}{\sinh 2 q}-0.192487\right)\left(1+e^{2 q}\right) \sin x$ & $u\left(x, t_{4}\right)=\left(\frac{q}{\sinh 2 q}-0.220991\right)\left(1+e^{2 q}\right) \sin x$ \\
$u_{5}(x)=\left(\frac{q}{\sinh 2 q}-0.208432\right)\left(1+e^{2 q}\right) \sin x$ & $u\left(x, t_{5}\right)=\left(\frac{q}{\sinh 2 q}-0.238651\right)\left(1+e^{2 q}\right) \sin x$ \\
$u_{6}(x)=\left(\frac{q}{\sinh 2 q}-0.216865\right)\left(1+e^{2 q}\right) \sin x$ & $u\left(x, t_{6}\right)=\left(\frac{q}{\sinh 2 q}-0.247617\right)\left(1+e^{2 q}\right) \sin x$ \\
$u_{7}(x)=\left(\frac{q}{\sinh 2 q}-0.219568\right)\left(1+e^{2 q}\right) \sin x$ & $u\left(x, t_{7}\right)=\left(\frac{q}{\sinh 2 q}-0.249988\right)\left(1+e^{2 q}\right) \sin x$ \\
$u_{8}(x)=\left(\frac{q}{\sinh 2 q}-0.217961\right)\left(1+e^{2 q}\right) \sin x$ & $u\left(x, t_{8}\right)=\left(\frac{q}{\sinh 2 q}-0.247432\right)\left(1+e^{2 q}\right) \sin x$ \\
$u_{9}(x)=\left(\frac{q}{\sinh 2 q}-0.213174\right)\left(1+e^{2 q}\right) \sin x$ & $u\left(x, t_{9}\right)=\left(\frac{q}{\sinh 2 q}-0.241271\right)\left(1+e^{2 q}\right) \sin x$ \\
$u_{10}(x)=\left(\frac{q}{\sinh 2 q}-0.206097\right)\left(1+e^{2 q}\right) \sin x$ & $u\left(x, t_{10}\right)=\left(\frac{q}{\sinh 2 q}-0.232544\right)\left(1+e^{2 q}\right) \sin x$ \\
\hline
\end{tabular}

Putting this into (5.20), we get a first-order linear differential equation in $T(t)$ which can be solved by calculating the integrating factor. Thus we have

$$
T(t)=\frac{2 \tau}{1-e^{-2 \tau}}-\left(1+e^{2 \tau}\right)\left(e^{-t}-e^{-2 t}\right) .
$$

Therefore, the exact solution is

$$
u(t, x)=\left[\frac{\tau}{\sinh 2 \tau}-\left(e^{-t}-e^{-2 t}\right)\right]\left(1+e^{2 \tau}\right) \sin x .
$$

Exact solutions for $t=t_{1}=0.1, \ldots, t=t_{10}=1$ are tabulated in Table 5.2.

On comparison of approximate solutions with exact solution of problem (5.1) at discrete values of variable $t$ in both cases, it is observed that they are very much similar to each other. It is also seen that for $\chi_{1} \neq \chi_{2}$ in $\mathscr{C}_{0}$, the corresponding solutions are different, which implies the existence of unique solution of (5.1).

As a second example we consider the same partial differential equation with a different nonlocal history condition:

$$
\begin{gathered}
\frac{\partial u}{\partial t}(t, x)-\frac{\partial^{2} u}{\partial x^{2}}(t, x)=u(t-\tau, x)-e^{-2 t}\left(1+e^{2 \tau}\right) \sin x, \quad t>0, x \in[0, \pi], \\
u(t, 0)=u(t, \pi)=0, \quad t>0, \\
\frac{1}{2 e^{2 \tau}} u(-\tau, x)+\frac{1}{2} u(0, x)=\sin x, \quad x \in[0, \pi] .
\end{gathered}
$$


192 Nonlocal history-valued delay differential equations

Table 5.3. Approximate and exact solutions for the second example $\left(\chi=e^{-2 t} \sin x\right)$.

\begin{tabular}{lc}
\hline Approximate solution & Exact solution \\
\hline$u_{1}(x)=0.834661 \sin x$ & $u\left(x, t_{1}\right)=0.818731 \sin x$ \\
$u_{2}(x)=0.697844 \sin x$ & $u\left(x, t_{2}\right)=0.670320 \sin x$ \\
$u_{3}(x)=0.584512 \sin x$ & $u\left(x, t_{3}\right)=0.548812 \sin x$ \\
$u_{4}(x)=0.490526 \sin x$ & $u\left(x, t_{4}\right)=0.449329 \sin x$ \\
$u_{5}(x)=0.412490 \sin x$ & $u\left(x, t_{5}\right)=0.367879 \sin x$ \\
$u_{6}(x)=0.347609 \sin x$ & $u\left(x, t_{6}\right)=0.301194 \sin x$ \\
$u_{7}(x)=0.293590 \sin x$ & $u\left(x, t_{7}\right)=0.246597 \sin x$ \\
$u_{8}(x)=0.248546 \sin x$ & $u\left(x, t_{8}\right)=0.201896 \sin x$ \\
$u_{9}(x)=0.210924 \sin x$ & $u\left(x, t_{9}\right)=0.165299 \sin x$ \\
$u_{10}(x)=0.179446 \sin x$ & $u\left(x, t_{10}\right)=0.135335 \sin x$ \\
\hline
\end{tabular}

An exact solution of (5.24) is

$$
u(t, x)=e^{-2 t} \sin x, \quad t \geq-\tau, x \in[0, \pi] .
$$

In a similar manner as before, for $\chi_{1} \in \mathscr{C}_{0}$ given by $\chi_{1}(t)(x)=e^{-2 t} \sin x$, approximations $u_{j}$ at discrete values $t_{j}, j=1,2, \ldots, 10$, of $t$ are

$$
u_{j}(x)=\frac{1}{(1+h)^{j}}\left[1-h e^{-2 h}\left(\frac{1-(1+h)^{j} e^{-2 j h}}{1-(1+h) e^{-2 h}}\right)\right] \sin x, \quad j=1,2, \ldots, 10 .
$$

Next, we choose $\chi_{2} \in \mathscr{C}_{0}$, such that $\chi_{2} \neq \chi_{1}$ satisfying the nonlocal history condition of (5.24), and given by

$$
\chi_{2}(t)(x)=\frac{2 e^{2 \tau}}{1+e^{2 \tau}} \sin x
$$

Following the similar steps of the previous example, here we get the approximate solutions

$$
u_{j}(x)=\left[\frac{2 e^{2 \tau}}{\left(1+e^{2 \tau}\right)^{2}}-\frac{h e^{-2 h}}{(1+h)^{j}}\left(\frac{1-(1+h)^{j} e^{-2 j h}}{1-(1+h) e^{-2 h}}\right)\right]\left(1+e^{2 \tau}\right) \sin x, \quad j=1,2, \ldots, 10,
$$

and the exact solution

$$
u(t, x)=\left[\frac{2 e^{2 \tau}}{\left(1+e^{2 \tau}\right)^{2}}-\left(e^{-t}-e^{-2 t}\right)\right]\left(1+e^{2 \tau}\right) \sin x, \quad j=1,2, \ldots, 10 .
$$

Putting $h=0.1$ in both cases, approximate as well as exact solutions are obtained. These approximate solutions $u_{j}, j=1,2, \ldots, 10$, corresponding to $\chi_{1}$ and $\chi_{2}$ along with their respective exact solutions are shown in Tables 5.3 and 5.4, respectively.

From these observations we arrive at a conclusion similar to the one of the previous example. 
Table 5.4. Approximate and exact solutions for the second example $\left(\chi=\left(2 e^{2 \tau} /\left(1+e^{2 \tau}\right)\right) \sin x\right)$.

\begin{tabular}{ll}
\hline Approximate solution & Exact solution \\
\hline$u_{1}(x)=\left(\frac{2 e^{2 q}}{\left(1+e^{2 q}\right)^{2}}-0.074430\right)\left(1+e^{2 q}\right) \sin x$ & $u\left(x, t_{1}\right)=\left(\frac{2 e^{2 q}}{\left(1+e^{2 q}\right)^{2}}-0.086106\right)\left(1+e^{2 q}\right) \sin x$ \\
$u_{2}(x)=\left(\frac{2 e^{2 q}}{\left(1+e^{2 q}\right)^{2}}-0.128602\right)\left(1+e^{2 q}\right) \sin x$ & $u\left(x, t_{2}\right)=\left(\frac{2 e^{2 q}}{\left(1+e^{2 q}\right)^{2}}-0.148411\right)\left(1+e^{2 q}\right) \sin x$ \\
$u_{3}(x)=\left(\frac{2 e^{2 q}}{\left(1+e^{2 q}\right)^{2}}-0.166803\right)\left(1+e^{2 q}\right) \sin x$ & $u\left(x, t_{3}\right)=\left(\frac{2 e^{2 q}}{\left(1+e^{2 q}\right)^{2}}-0.192007\right)\left(1+e^{2 q}\right) \sin x$ \\
$u_{4}(x)=\left(\frac{2 e^{2 q}}{\left(1+e^{2 q}\right)^{2}}-0.192487\right)\left(1+e^{2 q}\right) \sin x$ & $u\left(x, t_{4}\right)=\left(\frac{2 e^{2 q}}{\left(1+e^{2 q}\right)^{2}}-0.220991\right)\left(1+e^{2 q}\right) \sin x$ \\
$u_{5}(x)=\left(\frac{2 e^{2 q}}{\left(1+e^{2 q}\right)^{2}}-0.208432\right)\left(1+e^{2 q}\right) \sin x$ & $u\left(x, t_{5}\right)=\left(\frac{2 e^{2 q}}{\left(1+e^{2 q}\right)^{2}}-0.238651\right)\left(1+e^{2 q}\right) \sin x$ \\
$u_{6}(x)=\left(\frac{2 e^{2 q}}{\left(1+e^{2 q}\right)^{2}}-0.216865\right)\left(1+e^{2 q}\right) \sin x$ & $u\left(x, t_{6}\right)=\left(\frac{2 e^{2 q}}{\left(1+e^{2 q}\right)^{2}}-0.247617\right)\left(1+e^{2 q}\right) \sin x$ \\
$u_{7}(x)=\left(\frac{2 e^{2 q}}{\left(1+e^{2 q}\right)^{2}}-0.219568\right)\left(1+e^{2 q}\right) \sin x$ & $u\left(x, t_{7}\right)=\left(\frac{2 e^{2 q}}{\left(1+e^{2 q}\right)^{2}}-0.249988\right)\left(1+e^{2 q}\right) \sin x$ \\
$u_{8}(x)=\left(\frac{2 e^{2 q}}{\left(1+e^{2 q}\right)^{2}}-0.217961\right)\left(1+e^{2 q}\right) \sin x$ & $u\left(x, t_{8}\right)=\left(\frac{2 e^{2 q}}{\left(1+e^{2 q}\right)^{2}}-0.247432\right)\left(1+e^{2 q}\right) \sin x$ \\
$u_{9}(x)=\left(\frac{2 e^{2 q}}{\left(1+e^{2 q}\right)^{2}}-0.213174\right)\left(1+e^{2 q}\right) \sin x$ & $u\left(x, t_{9}\right)=\left(\frac{2 e^{2 q}}{\left(1+e^{2 q}\right)^{2}}-0.241271\right)\left(1+e^{2 q}\right) \sin x$ \\
$u_{10}(x)=\left(\frac{2 e^{2 q}}{\left(1+e^{2 q}\right)^{2}}-0.206097\right)\left(1+e^{2 q}\right) \sin x$ & $u\left(x, t_{10}\right)=\left(\frac{2 e^{2 q}}{\left(1+e^{2 q}\right)^{2}}-0.232544\right)\left(1+e^{2 q}\right) \sin x$
\end{tabular}

\section{Acknowledgments}

The authors would like to thank the National Board for Higher Mathematics for providing the financial support to carry out this work under its research project no. NBHM/ 2001/R\&D-II.

\section{References}

[1] S. Agarwal and D. Bahuguna, Existence and uniqueness of strong solutions to nonlinear nonlocal functional differential equations, Electron. J. Differential Equations (2004), no. 52, 1-9.

[2] - Method of semidiscretization in time to nonlinear retarded differential equations with nonlocal history conditions, Int. J. Math. Math. Sci. 2004 (2004), no. 37, 1943-1956.

[3] D. Bahuguna, Existence, uniqueness and regularity of solutions to semilinear nonlocal functional differential problems, Nonlinear Anal. Ser. A: Theory Methods 57 (2004), no. 7-8, 10211028.

[4] D. Bahuguna and V. Raghavendra, Application of Rothe's method to nonlinear integro-differential equations in Hilbert spaces, Nonlinear Anal. Ser. A: Theory Methods 23 (1994), no. 1, 7581.

[5] K. Balachandran and M. Chandrasekaran, Existence of solutions of a delay differential equation with nonlocal condition, Indian J. Pure Appl. Math. 27 (1996), no. 5, 443-449.

[6] L. Byszewski, Theorems about the existence and uniqueness of solutions of a semilinear evolution nonlocal Cauchy problem, J. Math. Anal. Appl. 162 (1991), no. 2, 494-505. 
[7] L. Byszewski and H. Akca, Existence of solutions of a semilinear functional-differential evolution nonlocal problem, Nonlinear Anal. Ser. A: Theory Methods 34 (1998), no. 1, 65-72.

[8] L. Byszewski and V. Lakshmikantham, Theorem about the existence and uniqueness of a solution of a nonlocal abstract Cauchy problem in a Banach space, Appl. Anal. 40 (1991), no. 1, 11-19.

[9] A. G. Kartsatos and M. E. Parrott, A method of lines for a nonlinear abstract functional evolution equation, Trans. Amer. Math. Soc. 286 (1984), no. 1, 73-89.

[10] A. G. Kartsatos and W. R. Zigler, Rothe's method and weak solutions of perturbed evolution equations in reflexive Banach spaces, Math. Ann. 219 (1976), no. 2, 159-166.

[11] Y. P. Lin and J. H. Liu, Semilinear integrodifferential equations with nonlocal Cauchy problem, Nonlinear Anal. Ser. A: Theory Methods 26 (1996), no. 5, 1023-1033.

[12] A. Pazy, Semigroups of Linear Operators and Applications to Partial Differential Equations, Applied Mathematical Sciences, vol. 44, Springer, New York, 1983.

S. Agarwal: Department of Mathematics, Indian Institute of Technology, Kanpur 208 016, India E-mail address: shrutiag@iitk.ac.in

D. Bahuguna: Department of Mathematics, Indian Institute of Technology, Kanpur 208 016, India E-mail address: dhiren@iitk.ac.in 


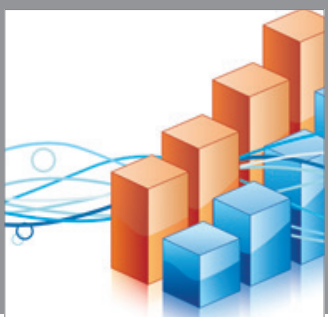

Advances in

Operations Research

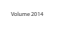

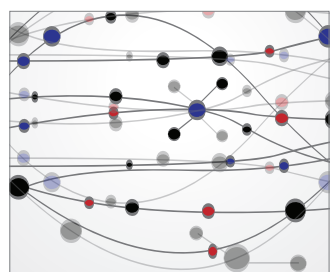

\section{The Scientific} World Journal
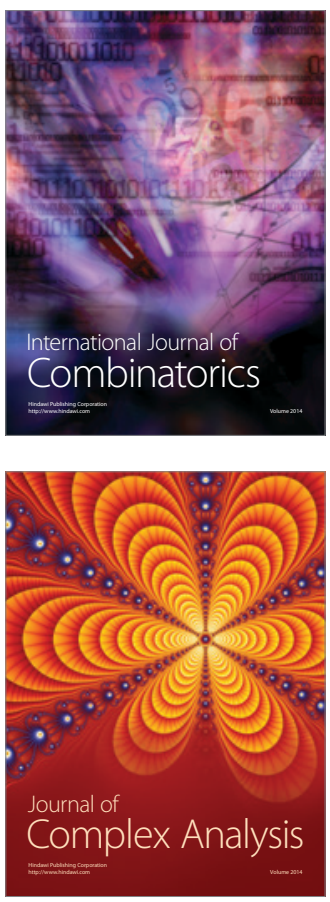

International Journal of

Mathematics and

Mathematical

Sciences
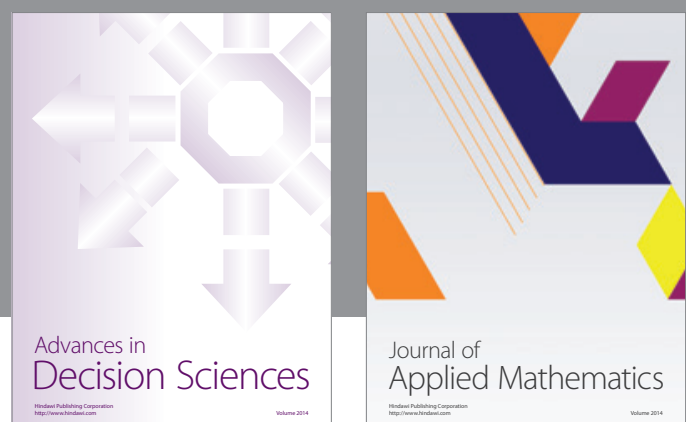

Journal of

Applied Mathematics
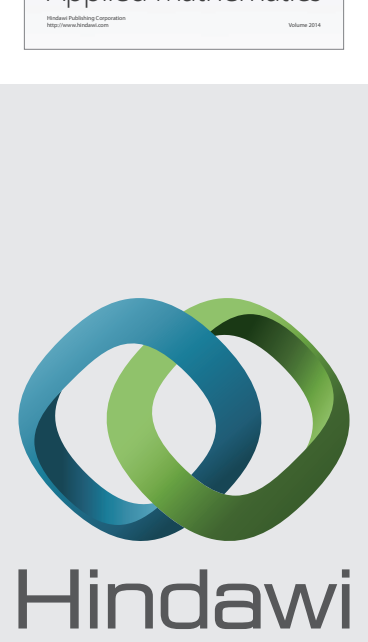

Submit your manuscripts at http://www.hindawi.com
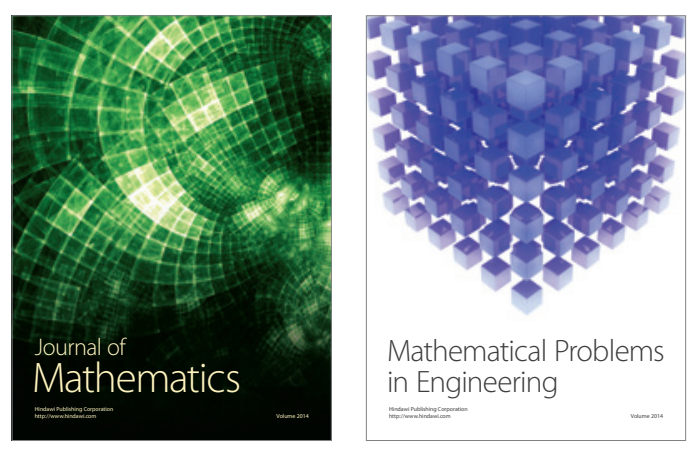

Mathematical Problems in Engineering
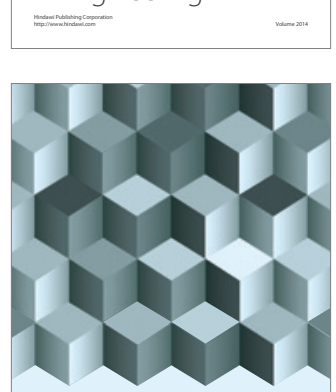

Journal of

Function Spaces
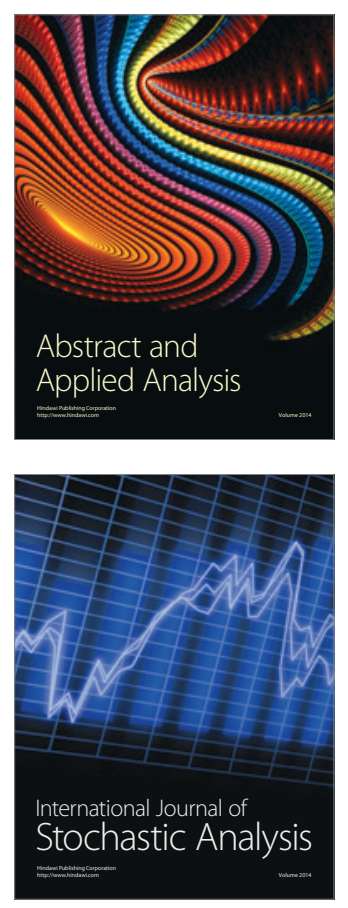

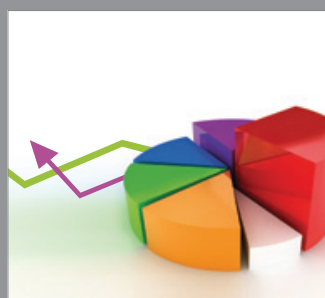

ournal of

Probability and Statistics

Promensencen
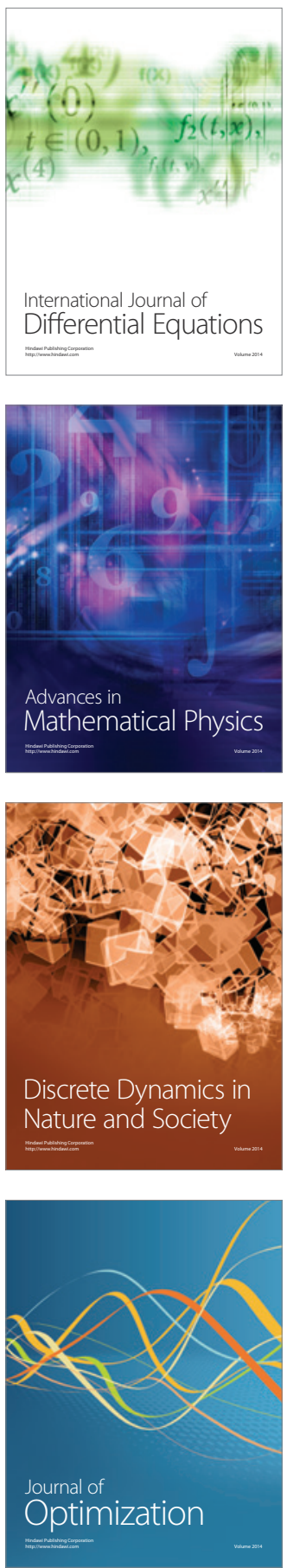